\title{
Methods for Essential Tremor Assessment: Acoustic Tremor Monitoring (ATM) and Rhythmic Spirals (RS) Methods
}

\author{
Emily Hart, Caroline Chow, Patricia Stan \& Daniel King \\ Department of Chemistry, Taylor University, Upland, IN \\ Students: emily_hart1@taylor.edu,cchow21490@gmail.com \\ Mentors: ptstan@taylor.edu,dnking@tayloru.edu
}

\begin{abstract}
There are several techniques of monitoring essential tremors, but there is not yet a standard method developed for the field. A quantitative way to track effects of medication and/or lifestyle treatment would be beneficial for future research in prevention or regression of essential tremors. The two methods evaluated are acoustic tremor monitoring (ATM) and rhythmic spirals (RS). The novel ATM measurement quantifies frequency and amplitude quickly and cost effectively. The tremor patient holds a microphone close to a speaker playing a single frequency tone. The Doppler Effect caused by the shaking microphone distorts the sound recording, and the encoded tremor information can be retrieved by using the Fast-Fourier Transform algorithm. The second method, RS, can be used by patients at home to measure frequency. The RS method is similar to the classic Archimedes spirals, but uses a different form and is timed which allows for the calculation of tremor frequency. The RS and ATM methods produce statistically similar frequency measurements, although ATM has greater precision.
\end{abstract}

\section{KEYWORDS}

Essential Tremors, Archimedes Spiral, Accelerometry, Spiral Analysis, Acoustic Tremor Monitoring, Rhythmic Spirals.

\section{INTRODUCTION}

Essential tremor (ET) is a condition that affects millions of Americans and for which there does not currently exist a standard quantitative clinical assessment method. The progression of the disorder and its correlation to other neurodegenerative disorders are poorly understood and, consequently, patient care and research could be dramatically improved with the development of a simple, effective quantitative assessment method. Tremors are defined as repetitive, rhythmic, involuntary, oscillations of a body part, and are the most common of all movement disorders., ${ }^{1,2,3}$ Two primary approaches have been used for classifying tremors. One system focuses on when the tremors occur. If a tremor is produced when the affected part is relaxed it is called a static tremor. ${ }^{1}$ If a tremor starts during a voluntary muscular contraction it is called an action tremor. Action tremors can be further broken down into more specific classifications including postural, isometric, kinetic, task-specific, and intentional tremors. ${ }^{1}$ The second system of classification focuses on the underlying cause of the tremor, either physiological or pathological., ${ }^{1,2}$ Physiological tremors happen in all humans and can be seen or unseen and often become more visible within specific situations, like being tired or hungry, and with age. ${ }^{1,2}$ Pathological tremors are associated with neurological disorders and include cerebellar, psychogenic, orthostatic, neuropathic, parkinsonian, and essential tremors. ${ }^{2}$ Despite the fact that many ET cases do not seek medical help, ${ }^{4}$ there is still an estimated 10 million Americans diagnosed with essential tremors which makes it the most common pathological tremor. ${ }^{5}$

Recent studies about the progression of ET indicate subjects experience a 3-5\% increase in the 
Total Tremor Scale (total number of tremor occurrences) or a 12\% increase in the Tremor Rating Scale (tremor severity) each year. ${ }^{6,7}$ Both scales are based on the visible observation of tremor symptoms. Although both studies utilized expert movement disorder neurologists to make the observations, inconsistencies were found between the calculated disease progression rates of the two methods. Additionally, studies have found ET patients to be at increased risk for other diseases or disabilities including cognitive problems (speech and memory), anxiety, depression, social phobia, balance, hearing issues, Parkinson's disease and dementia. ${ }^{4}$ As a result of these new findings, long term studies of ET patients are needed, utilizing a standard methodology to obtain consistent and quantitative measurements of the progression or regression of the disease.

Many techniques have been developed to measure the frequency and amplitude of tremors. Many physicians employ different qualitative severity tests, including questionnaires, that use numbered scales to rank the severity or intensity of tremors. ${ }^{1,2}$ Drawings like the Archimedes spiral are also being used to watch the progression of the tremor ${ }^{3,8}$ and to compare with other previously diagnosed patients. ${ }^{2}$ More sophisticated quantitative ways of measuring frequency and amplitude electronically are being created; a few methods include accelerometry, electromyography (EMG), magnetic tracker system, active optical markers, gyroscopes, spirography, and spiral analysis. ${ }^{1,2,3,9}$ However, despite the variety of qualitative methods and newer quantitative methods, physicians have yet to embrace a standard assessment method, perhaps because the qualitative tests are too subjective and the quantitative methods are too inaccessibly high tech to be clinically useful.

In an attempt to develop two complimentary methods, one that could easily be used by patients at home and one more robust method for clinicians, to measure tremor frequency and monitor tremor progression over time, we report here the novel Acoustic Tremor Monitoring and Rhythmic Spiral methods. The Acoustic Tremor Monitoring (ATM) method is a fast, easy, non-invasive method to measure frequency and observe the amplitude of a tremor. ATM uses sound waves that are distorted by a tremor and visualized with the Fast Fourier Transform algorithm. The Rhythmic Spiral (RS) method is an adaptation of the classic Archimedes spiral exercise already in use, performed at a specific rate so as to reveal tremor frequency information, and would be simple for patients to perform at home to estimate their tremor frequency.

\section{METHODS}

IRB approval was granted for this work. The pilot study participant was provided an Informed Consent, and both written and oral description of the project. The subject was asked to hold a microphone several times with each hand to collect the ATM signal and to complete the RS writing assessment several times to collect the RS data. The total involvement of the subject required less than two hours.

\section{Acoustic Tremor Monitoring: Clinical assessment}

The Acoustic Tremor Monitoring method can be used to analyze tremor frequency and amplitude from the Doppler distortion imparted into a sound recording when the microphone is held by a tremor subject (Figure 1). An inexpensive desktop microphone is used to record a sound file, held at approximately 6 inches from the $2000 \mathrm{~Hz}$ tone source. The Fast Fourier Transform (FFT) algorithm, a standard function of data processing software package Origin9.0 (OriginLab) is used to convert the sound wave to a frequency spectrum with respect to the magnitude of the signal. Initially, the microphone was exposed to a variety of conditions of mechanical oscillations that mimic hand tremors. These samples were recorded in WMA files and then converted into WAV files through a free online converter ${ }^{10}$ so that they could be processed by the Origin 9.0 software program. Frequency spectra are normalized to the most intense frequency $(2000 \mathrm{~Hz})$ and displayed as 100 percent relative intensity. 


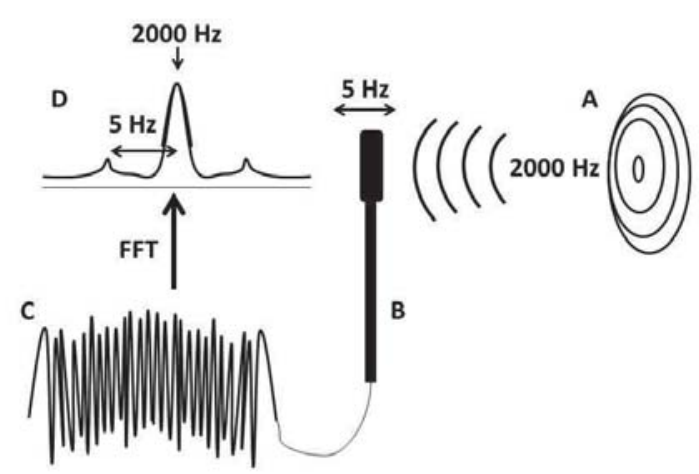

Figure 1. A schematic representation of the experimental setup is shown. (A) A $2000 \mathrm{~Hz}$ tone is played and recorded by a standard computer microphone (B) while being held by a subject. The resulting time-domain sound file (C) is processed by Fourier transform to yield frequency-domain spectrum (D). The tremor frequency and source tone frequency information is revealed in the frequency spectrum.

\section{Effect of Tremor Frequency on ATM Signal}

To evaluate the effect of tremor frequency on the ATM signal, a Gyrotory Water Bath Shaker, Model G76, was used with a microphone taped to one of the shaking bars. The shaker has a variable speed dial that allowed for varying speed and, consequently, frequency. A high pitch tone at 2000 $\mathrm{Hz}$ was played approximately 6 inches from the microphone, and recorded for about 5 seconds at each different speed setting, 1 to 5 .

\section{Effect of Tremor Amplitude on ATM Signal}

To evaluate the effect of tremor amplitude, or distance the microphone travels, on the ATM signal, the microphone was oscillated for varying distances but at a fixed frequency. Paths of half an inch, one inch, two inches, and three inches were marked on a piece of paper. A digital metronome was played at 208 beats per minute into a pair of headphones. With the high pitch tone $(2000 \mathrm{~Hz})$ being played approximately 6 inches from the microphone and while listening to the metronome through headphones, the microphone was manually oscillated the designated distances to the constant beat of the metronome. The signal was recorded for about 5 seconds.

\section{Rhythmic Spiral: Home assessment}

The Rhythmic Spiral method attempts to control the speed at which the traditional hand written Archimedes spiral is performed, providing a known and consistent rate, in order to impart meaningful tremor frequency information into the produced drawing. The spiral pattern used consists of 6 loops drawn side by side to make the shape of a spring (Figure 2). With a metronome playing at 40 beats per minute, the subject is asked to trace the spring while trying to hit the bottom of each loop on the beat. When finished the entire pattern should be completed in 9 seconds. The imperfections (zigzags) visible in the trace are the result of the tremor, and each tremor event results in one zigzag motion. Therefore, each zigzag (right - left pair) is counted as a single event. Then, the number of observed events is divided by 9 seconds to produce a solid estimate for the frequency of the tremor. The pattern can be repeated several times to calculate an average tremor frequency.

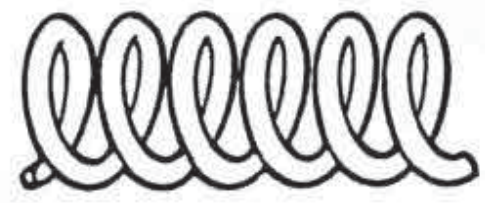

Figure 2. A sample spiral template pattern is shown. 


\section{RESULTS AND DISCUSSION:}

ATM

Acoustic Tremor Monitoring (ATM) takes advantage of the Doppler distortion that occurs when a monochromatic tone is recorded by a microphone that is shaking. Just like the higher and lower pitches heard when a police car and siren pass by quickly, the ATM picks up the played tone at its original frequency and at the original frequency plus and minus the frequency of the shaking. In an initial attempt to assess if the ATM method is sensitive enough to observe the shaking hand of an ET subject, a volunteer who presents with moderate ET in the right arm (dominant) but symptom free in the left arm (non-dominant) was selected. ET often develops first in the dominant hand, as it did for this subject. A 5 second recording of the monochromatic $2000 \mathrm{~Hz}$ tone with the microphone sitting on the table was collected to serve as a blank. A 5 second negative control was collected with the subject holding the microphone in the symptom free left hand. Then, a similar recording with the subject's ET affected right was collected. The frequency spectra are shown in Figure 3. As expected, two symmetrical side bands appeared in the tremor signal, likely resulting from the Doppler shift in the recorded frequencies. The correlation of these side bands to the frequency and amplitude of the tremor is evaluated below. Encouragingly, the negative control strongly resembles the blank and is easily distinguishable from the ET affected hand.

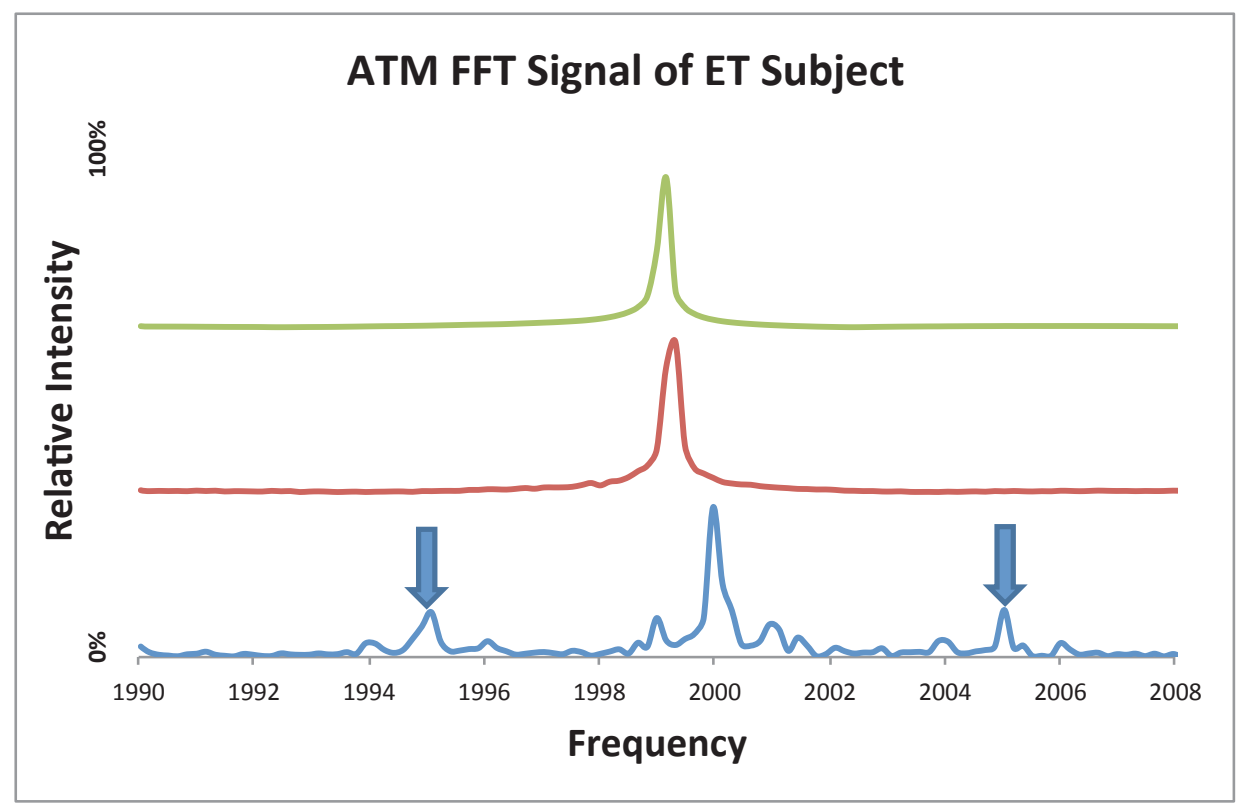

Figure 3. ATM signals for the blank $2000 \mathrm{~Hz}$ signal (green), the negative control with the subject's asymptomatic left hand (red), and the sample with the subject's symptomatic right hand (blue) are shown. The arrows point to the Doppler shifted side bands that are indicative of a shaking microphone.

\section{Effect of Tremor Frequency on ATM Signal}

To assess the effect of tremor frequency, the microphone was attached to a water bath shaker, and $2000 \mathrm{~Hz}$ recordings were made at five different speed settings. As the speed setting was increased, and consequently frequency, the Doppler peaks grew farther apart from one another. Predicting that the distance between the principle peak and each Doppler peak is the frequency at which the microphone oscillates, ten oscillations were observed and timed to determine the actual shaker frequency. Averaging four trials at each speed, the frequency of the shaker speeds 1-5 were determined to be $0.41,1.12,1.82,2.59$, and $3.37 \mathrm{~Hz}$, respectively. The frequencies determined by the ATM splitting distances were very similar to the manually determined frequencies of the shaker 
(NA, 1.08, 1.75, 2.58, and $3.39 \mathrm{~Hz}$ ). The similarity is statistically significant ( $\mathrm{p}<0.05$ ), and when plotted against each other correlate with an $\mathrm{R}^{2}$ value of 0.9995 . The ATM signals for speeds $2-5$ are displayed in Figure 4. It is noteworthy that the Doppler shifted peaks were not resolved for the slowest frequency $(0.41 \mathrm{~Hz})$. The results suggest an apparent lower frequency limit for the ATM method; however, it is far slower than the typical ET frequencies, 3-12 Hz. The slight shifting of the spectra along the frequency axis between trials appears to be a result of the slight inconsistency of the source tone. The fact that the side bands shift proportionally with the source peak, $2000 \mathrm{~Hz}$, suggests that the measured tremor frequency, the distance between the source peak and the side bands, will be unaffected.

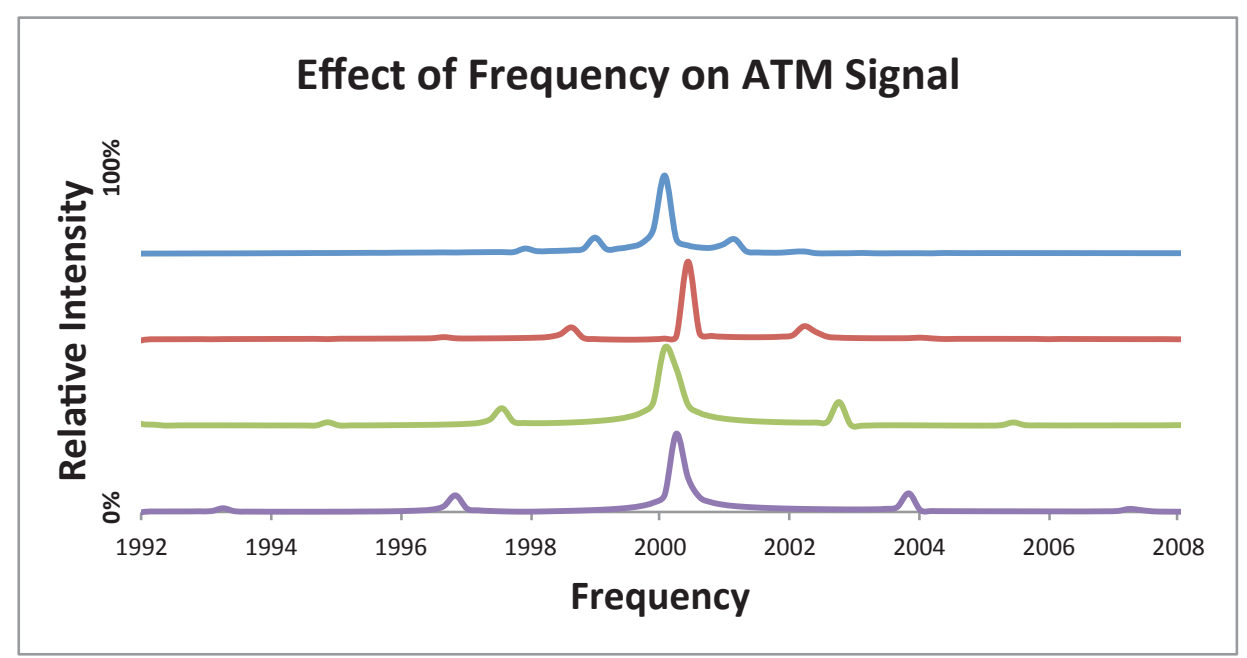

Figure 4. Individual, normalized ATM signals at various mechanical shaker speed settings are shown: speed $2=1.08 \mathrm{~Hz}$ (blue), speed $3=1.75 \mathrm{~Hz}$ (red), speed $4=2.58 \mathrm{~Hz}$ (green), and speed $5=3.39 \mathrm{~Hz}$ (purple).

\section{Effect of Tremor Amplitude on ATM Signal}

To assess the effect of tremor amplitude on the ATM signal, a set of tests were performed where the distance the microphone traveled was varied while holding the oscillation frequency constant. Using a metronome with headphones to maintain a constant frequency, the microphone was oscillated through a set of various distances marked on a table to simulate a variety of tremor amplitudes at 208 beats per minute. Manually translating the microphone through one oscillation (back and forth through the measured distances) for each beat produced a frequency of $3.47 \mathrm{~Hz}$. The ATM data collected for these trials produced an average measured frequency of $3.53 \mathrm{~Hz}(1.7 \%$ difference). The Doppler spacing remained constant for these trials indicating that a consistent oscillation frequency was maintained. The Doppler shifted peaks' heights, however, varied significantly with amplitude. As the amplitude of the oscillations increased, the height of the Doppler shifted peaks increased systematically, although not linearly (linear R $2=0.961$, exponential R2 $=0.9986$ ). The Doppler shifted peak heights for the three distances (1,2, and 3 inches) were $4.9 \%, 12.2 \%$, and $27.5 \%$ of the principle $2000 \mathrm{~Hz}$ peak, respectively (Figure 5). Although, this trend was expected to be linear, the deviation from linearity may be a result of slight inconsistencies in manually translating the microphone specified distances. 


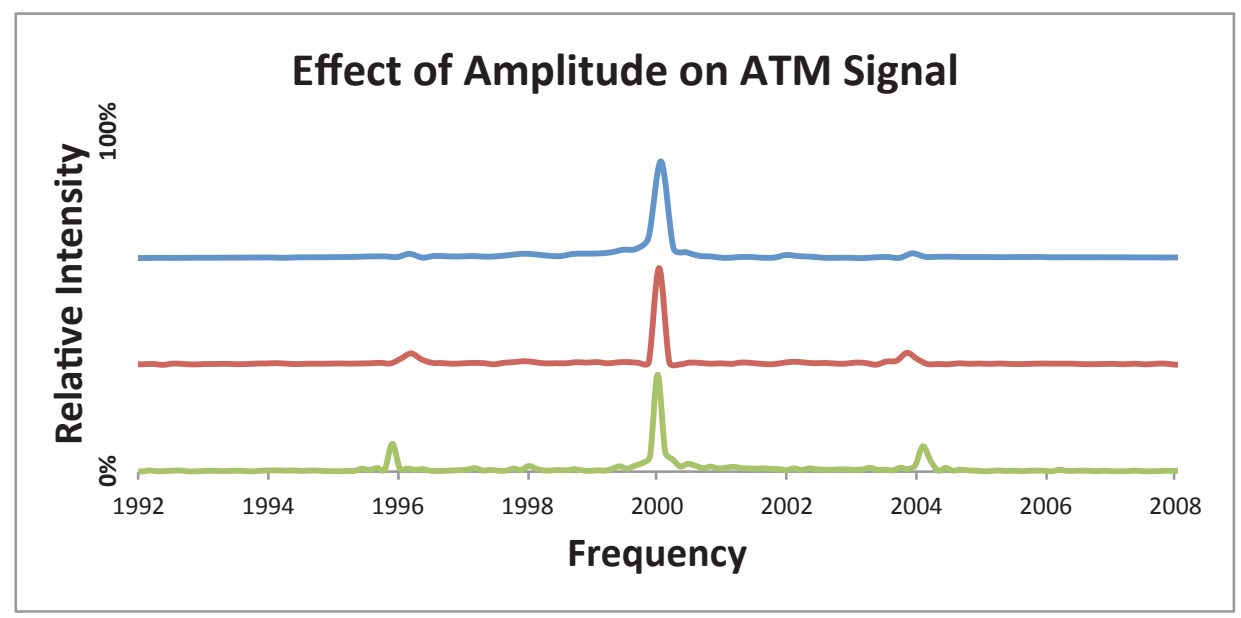

Figure 5. Normalized ATM signals for $3.47 \mathrm{~Hz}$ oscillations over distances of 1 inch (blue), 2 inches (red), and 3 inches (green) are shown.

\section{Rhythmic Spiral Home Assessment}

In addition to the clinical ATM method, the classic Archimedes spiral was adapted to provide tremor patients with a cheap and simple way to assess their tremor frequency at home. Once a patient becomes comfortable tracing the spiral pattern to a beat and counting the tremor features, the frequency of an essential tremor can be quickly measured. The same ET volunteer who participated in testing the ATM methods, with moderate essential tremor in the right hand, also performed the RS test. Although the RS test could in principle be performed on a non-dominant hand, most subjects will likely lack the dexterity to do so. Fortuitously for this analysis, ET is often more significantly developed and most inconvenient in the dominant hand. Figure 6 shows a sample RS test with each zigzag pair marked as a single event. The total number of tremor events are counted and divided by 9 to calculate the tremor frequency estimate. It is reasonable to expect that the amplitude may correlate to the size of the zigzags, but we have yet to devise a method for evaluating this hypothesis. The RS test was performed 19 times on the subject to yield an average observed tremor frequency of $4.7 \mathrm{~Hz}$ (standard deviation $= \pm 0.43 \mathrm{~Hz}$ ). The average ATM frequency measurements for the same subject were $5.0 \mathrm{~Hz}$ (standard deviation $= \pm 0.11 \mathrm{~Hz}$ ). Both the RS and the ATM method are compared in Figure 7 below. The resulting frequencies from both methods agree quite well, with the ATM method showing greater precision. Both methods were sensitive enough to evaluate this moderate essential tremor subject.

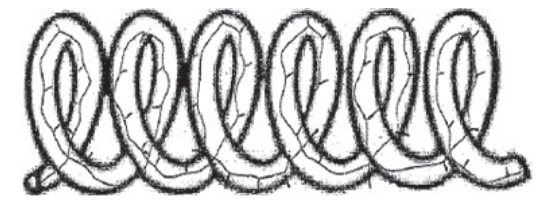

Figure 6. A sample RS sample from an ET subject is shown. The RS trace shows the typical zigzag pattern, where each zigzag pair is indicative of a single tremor event. The total events divided by the total time, 9 sec., yields the tremor frequency. 


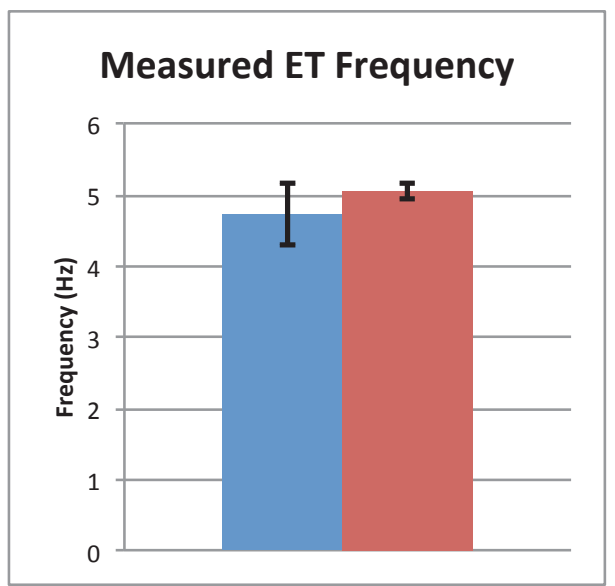

Figure 7. The average measured frequency from $19 \mathrm{RS}$ tests, $4.7 \mathrm{~Hz}$, and standard deviation, $\pm 0.43 \mathrm{~Hz}$, for an ET subject is shown in blue. The average measured frequency from 4 ATM tests, $5.0 \mathrm{~Hz}$, and standard deviation, $\pm 0.11 \mathrm{~Hz}$, for the same ET subject is shown in red.

\section{CONCLUSIONS AND FUTURE WORK}

The ATM and RS methods are sufficiently accurate, precise, and accessible to warrant further evaluation as to their usefulness for the routine monitoring of essential tremors and potentially for diagnosis. ATM may be capable of accurately measuring tremor frequency and observing the change in amplitude of a tremor with minimal technological requirements. Although ATM has been cast here as a potential clinical method, it is reasonable to imagine patients collecting the ATM recordings on their own at home and electronically sending the file to a clinician for evaluation. The ATM method has the distinct advantage over RS in that its frequency measurements are more precise and it can quantitatively evaluate amplitude. The RS method is a completely low tech variation of what many ET patients are already doing, and can be used to easily keep track of the frequency at home. The RS method provides reliable frequency measurements and may prove more accessible to the clinical community because of its similarity to the Archimedes spirals currently being used. Although only tested with one subject, the tremor frequencies measured by ATM and RS are consistent and complementary warranting their further evaluation in a larger ET population.

Future collaborations with physicians specializing in ET to study a larger ET population will be necessary to evaluate the full potential of the ATM and RS methods. A large ET population should be evaluated by ATM and the results compared to the subjective scale assessments performed by the clinicians. Finding tangible correlations between ATM frequency and amplitude values and physician assessment scales would not only substantiate the ATM method but could also provide valuable insight into the progression of ET if patients are monitored over a long period of time. Additionally, the same ET patients would perform RS tests on their own at home to allow for not only a large scale comparison of RS and ATM results, but also the evaluation of the precision and accuracy patients are able to achieve performing the test at home.

For a given ET patient, tremors may fluctuate significantly for reasons such as fatigue, hunger, and caffeine ingestion. Consequently, having annual qualitative clinical assessments may not only be quite subjective, but will be susceptible to the temporal variations of the tremor itself. Simply put, patients can have good days and bad days. Similarly, because of these same natural variations it is difficult for patients to assess the effectiveness of a newly prescribed treatment, like alcohol or beta-blockers, since so many things can affect the tremor symptoms. A quantitative assessment tool that could be used routinely in order to yield reliable and meaningful average tremor measures could dramatically improve the specificity of the care patients could receive, greatly increase the chances for researchers to find meaningful correlations between ET progression and the development of other neurological and psychological conditions, and empower ET patients who generally feel helpless and hopeless with regard to their ET progression. 


\section{REFERENCES}

[1] Mansur, P.H.G., Cury, L.K.P., Andrade, A.O., Pereira, A.A., Miotto, G.A.A., Soares, A.B., Naves, E.L.M. (2007) A Review on Techniques for Tremor Recording and Quantification Biomedical Engineering 35, 343-362.

[2] Andrade, A. O., Pereira, A. A., Soares, M. F., de Almeida, G. L. C., Paixão, A. P. S., Fenelon, S. B., \& Dionisio, V. C. (2012). Human Tremor: Origins, Detection and Quantification, in Practical Applications in Biomedical Engineering (Andrade, A. O., Pereira, A. A., Naves, E. L. M., Soares, A. B., Ed.) pp 3-24, InTech.

[3] Almeida, M. F. S., Cavalheiro, G. L., Pereira, A. A., \& Andrade, A. O. (2010). Investigation of age-related changes in physiological kinetic tremor. Annals of biomedical engineering, 38 (11), 34233439.

[4] Louis, E. D., \& Okun, M. S. (2011). It is time to remove the 'benign' from the essential tremor label. Parkinsonism \& related disorders, 17 (7), 516-520.

[5] Facts about Essential Tremor, International Essential Tremor Foundation, http://www.essentialtremor.org/wp-content/uploads/2013/07/FactSheet012013.pdf (accessed Oct 2014)

[6] Putzke, J. D., Whaley, N. R., Baba, Y., Wszolek, Z. K., \& Uitti, R. J. (2006). Essential tremor: predictors of disease progression in a clinical cohort. Journal of Neurology, Neurosurgery \& Psychiatry, 77 (11), 1235-1237.

[7] Louis, E. D., Agnew, A., Gillman, A., Gerbin, M., \& Viner, A. S. (2011). Estimating annual rate of decline: prospective, longitudinal data on arm tremor severity in two groups of essential tremor cases. Journal of Neurology, Neurosurgery \& Psychiatry, 82 (7), 761-765.

[8] Ellias, S. A. (1993). Essential tremor. Rhode Island Medicine, 76, 563-566.

[9] Diagnostic Tests of Motor Function, CMPL, Columbia University Medical Center, Department of Neurology, http://cmpl.columbia.edu/diagnostic.html\#top (accessed Oct 2014).

[10] Burkard, J., Online Audio Converter, http://media.io/ (accessed Oct 2014).

\section{ABOUT THE STUDENT AUTHORS}

Emily Hart is a senior at Taylor University working towards a BA degree in Chemistry. Caroline Chow was a 2012 Taylor University graduate and is now a chemistry teacher at Jac-Cen-Del High School, Osgood, IN.

\section{PRESS SUMMARY}

We developed two methods to assess essential tremors: acoustic tremor monitoring (ATM) and rhythmic spirals (RS) methods. These complementary methods are easy, non-invasive techniques that are likely to measure essential tremors quickly and inexpensively. ATM uses sound waves to monitor the frequency and amplitude of the tremor. RS is an at home method adapted from Archimedes spirals which gives a quick estimate of a tremor's frequency. 